\title{
LUNA: hydrogen, helium and carbon burning under Gran Sasso
}

\author{
Carlo Broggini ${ }^{* \dagger}$ \\ INFN-Sezione di Padova \\ E-mail: broggini@pd.infn.it
}

\begin{abstract}
One of the main ingredients of nuclear astrophysics is the cross section of the thermonuclear reactions which power the stars and synthesize the chemical elements in the Universe. Deep underground in the Gran Sasso Laboratory the cross section of the key reactions of the protonproton chain and of the Carbon-Nitrogen-Oxygen (CNO) cycle have been measured right down to the energies of astrophysical interest. The main results obtained during the 'solar' phase of LUNA are reviewed and their influence on our understanding of the properties of the neutrino and of the Sun is discussed. We then describe the current LUNA program devoted to the study of the nucleosynthesis of the light elements in AGB stars, Classical Novae and at the time of the big bang. Finally, the future of LUNA with the new 3.5 MV accelerator is outlined. This single-ended accelerator will be installed under Gran Sasso in spring 2018. It will provide hydrogen, helium and carbon (also doubly ionized) high current beams and it will be devoted to the study of helium and carbon burning in stars.
\end{abstract}

Frontier Research in Astrophysics - II

23-28 May 2016

Mondello (Palermo), Italy

* Speaker.

${ }^{\dagger}$ for the LUNA Collaboration 


\section{Introduction}

Stars are not perfect and eternal bodies as believed by the ancient philosophers. On the contrary, gravity triggers the birth of a star which then works as a fusion reactor to finally die out in a quiet or violent way, depending on its initial mass. As a matter of fact, only hydrogen, helium and lithium are synthesized in the first minutes after the big-bang. All the other elements in the Universe are produced in the thermonuclear reactions taking place inside the 'cauldrons' active in the cosmos, i.e. the stars. Nuclear astrophysics studies the reactions which transmute the chemical elements and provide the energy to the stars. In particular, the knowledge of the fusion reaction cross-section at the stellar energies is the heart of nuclear astrophysics.

The reaction occurs in the hot plasma of a star, with temperatures in the range of tens to hundreds of millions degrees, inside an energy window, the Gamow peak, which is far below the Coulomb energy arising from the repulsion between nuclei. At energies below the Gamow peak the cross section is too small whereas at higher energies the nuclei are too few. In this region the cross section is given by:

$$
\sigma(E)=\frac{S(E)}{E} \exp (-2 \pi \eta)
$$

where $S(E)$ is the astrophysical factor (which contains the nuclear physics information) and $\eta$ is given by $2 \pi \eta=31.29 Z_{1} Z_{2}(\mu / E)^{1 / 2} . Z_{1}$ and $Z_{2}$ are the nuclear charges of the interacting particles, $\mu$ is the reduced mass (in units of amu), and $E$ is the center of mass energy (in units of keV).

At low energies the cross sections are extremely small, because of the small probability to go through the Coulomb barrier. Such smallness makes the star life-time of the length we observe, but it also makes impossible the direct measurement in the laboratory. The rate of the reactions, characterized by a typical energy release of a few $\mathrm{MeV}$, is too low, down to a few events per year, in order to stand out from the laboratory background. Instead, the observed energy dependence of the cross-section at high energies is extrapolated to the low energy region, leading to substantial uncertainties. LUNA, Laboratory for Underground Nuclear Astrophysics, started twenty five years ago to run nuclear physics experiments in an extremely low-background environment, the Gran Sasso Laboratory (LNGS), to reproduce in the laboratory what Nature makes inside the stars [1, 2].

\section{Accelerators under Gran Sasso}

Two electrostatic accelerators able to deliver hydrogen or helium beams have been installed in LUNA: first a compact $50 \mathrm{kV}$ "home made" machine [3] and then, in the year 2000, a commercial $400 \mathrm{kV}$ one [4]. Common features of the two accelerators are the high beam current, the long term stability and the precise beam energy determination.

In particular, the $400 \mathrm{kV}$ accelerator is embedded in a tank, a cylinder of $0.9 \mathrm{~m}$ diameter and 2.8 $\mathrm{m}$ long, filled with an insulating mixture of $\mathrm{N} 2 / \mathrm{CO} 2$ gas at 20 bar. The high voltage is generated by an inline Cockcroft-Walton power supply located inside the tank. The radio frequency ion source directly mounted on the accelerator tube can provide beams of hydrogen or $\mathrm{He}^{+}$over a continuous operating time of 40 days. The ions can then be sent into one of two different, parallel beam lines (fig.1), allowing the installation of two different target setups. In the energy range between 150 and $400 \mathrm{keV}$, the accelerator can provide up to $0.5 \mathrm{~mA}$ of hydrogen and $0.25 \mathrm{~mA}$ of helium at the 


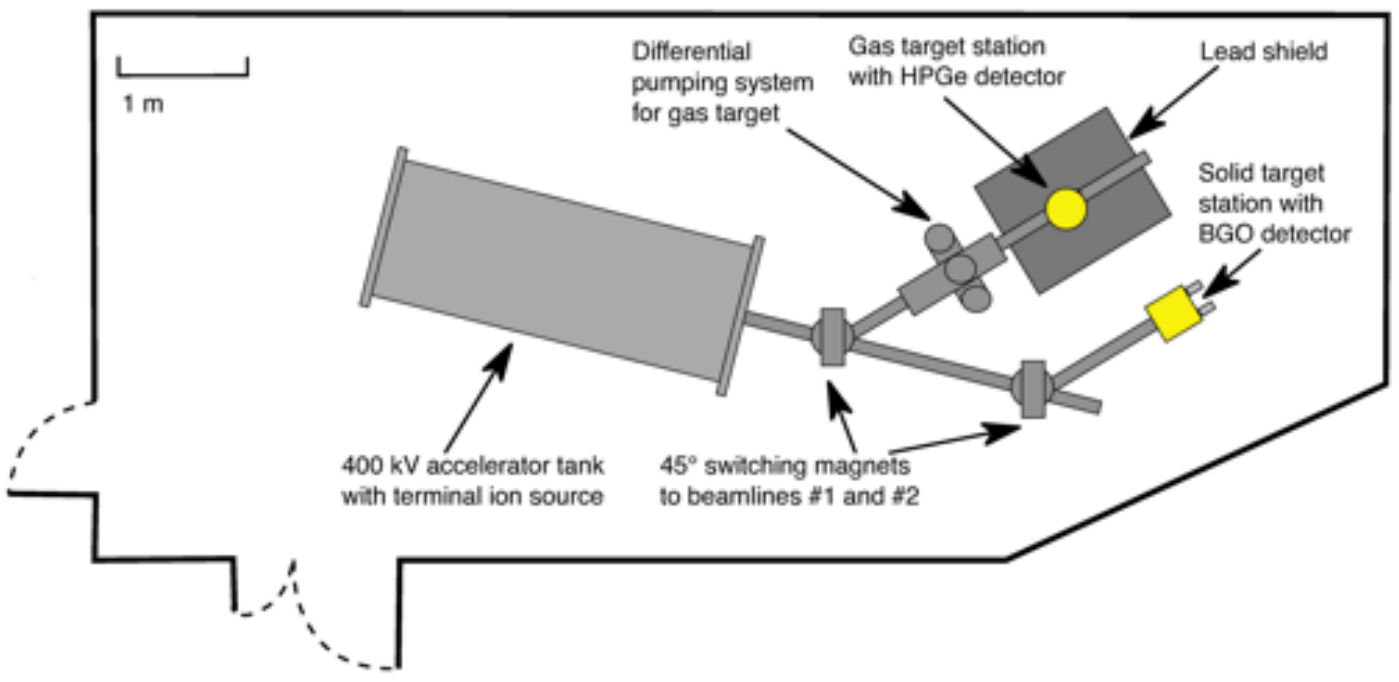

Figure 1: The LUNA $400 \mathrm{kV}$ accelerator with the two beam lines devoted to gas and solid target experiments, respectively.

target stations, with $0.3 \mathrm{keV}$ accuracy on the beam energy, $100 \mathrm{eV}$ energy spread, and $5 \mathrm{eV}$ per hour long-term stability.

The dolomite rock of Gran Sasso provides a natural shielding equivalent to at least 3800 meters of water which reduces the muon and neutron fluxes by a factor $10^{6}$ and $10^{3}$, respectively. The neutron flux underground is mainly due to $(\alpha, n)$ reactions in the rock, with the alpha particles coming from the ${ }^{238} \mathrm{U}$ and ${ }^{232} \mathrm{Th}$ decay chains. Finally, the activity due to Radon from the rock is suppressed down to the level of few tens of Bequerel $/ \mathrm{m}^{3}$ thanks to frequent air volume exchanges (once every 3.5 hours) [5].

\section{Hydrogen burning in the Sun}

The first phase of LUNA, the solar phase, has been driven by the solar neutrino problem [6]. In particular, the initial activity has been focused on the ${ }^{3} \mathrm{He}\left({ }^{3} \mathrm{He}, 2 \mathrm{p}\right){ }^{4} \mathrm{He}$ cross section measurement within the solar Gamow peak (15-27 keV). Such a reaction is a key one of the hydrogen burning proton-proton chain, which is responsible for more than $99 \%$ of the solar luminosity. A resonance in its cross section at the thermal energy of the Sun was suggested long time ago to explain the observed ${ }^{8} \mathrm{~B}$ solar neutrino flux. As a matter of fact, such a resonance would decrease the relative contribution of the alternative reaction ${ }^{3} \mathrm{He}(\alpha, \gamma){ }^{7} \mathrm{Be}$, which generates the branch responsible for ${ }^{7} \mathrm{Be}$ and ${ }^{8} \mathrm{~B}$ neutrino production in the Sun.

The experimental set-up was made of eight $1 \mathrm{~mm}$ thick silicon detectors of $5 \times 5 \mathrm{~cm}^{2}$ area placed around the beam inside the windowless target chamber filled with ${ }^{3} \mathrm{He}$ at the pressure of $0.5 \mathrm{mbar}$. The simultaneous detection of two protons has been the signature which unambiguously identified a ${ }^{3} \mathrm{He}\left({ }^{3} \mathrm{He}, 2 \mathrm{p}\right){ }^{4} \mathrm{He}$ fusion reaction. Fig.2 shows the results from LUNA [7] together with higher energy measurements $[8,9,10]$. For the first time a nuclear reaction has been measured in the laboratory at the energy occurring in a star. In particular, at the lowest energy of $16.5 \mathrm{keV}$ the 


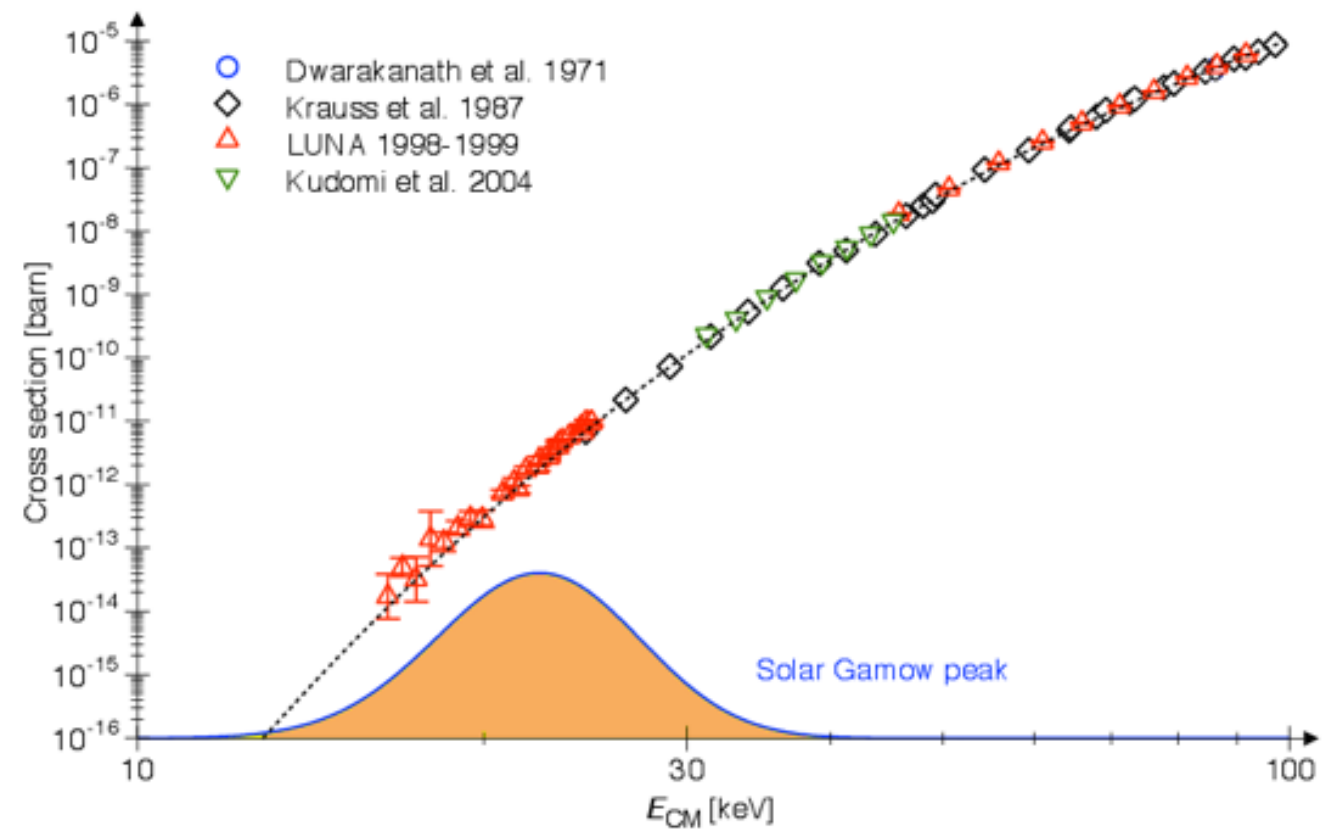

Figure 2: The cross section of ${ }^{3} \mathrm{He}\left({ }^{3} \mathrm{He}, 2 p\right)^{4} \mathrm{He}$ as function of energy.

cross section is 0.02 pbarn, which corresponds to a rate of about 2 events/month, rather low even for the "silent" experiments of underground physics. No narrow resonance has been found and, as a consequence, the astrophysical solution of the ${ }^{8} \mathrm{~B}$ and ${ }^{7} \mathrm{Be}$ solar neutrino problem based on its existence has been definitely ruled out.

${ }^{3} \mathrm{He}(\alpha, \gamma){ }^{7} \mathrm{Be}$, the competing reaction for ${ }^{3} \mathrm{He}$ burning, has also been measured by LUNA both by detecting the prompt $\gamma$ rays and by counting the ${ }^{7} \mathrm{Be}$ nuclei from their decay. The two different methods gave the same result within the total error of $4 \%$ [11].

\subsection{The CNO neutrinos from the Sun}

${ }^{14} \mathrm{~N}(\mathrm{p}, \gamma){ }^{15} \mathrm{O}$ is the slowest reaction of the first $\mathrm{CNO}$ cycle and it rules its energy production rate. In particular, it is the key reaction to predict the ${ }^{13} \mathrm{~N}$ and ${ }^{15} \mathrm{O}$ solar neutrino flux, which depends almost linearly on its cross section.

In the first phase of the LUNA study, data have been obtained down to $119 \mathrm{keV}$ energy with solid targets of TiN and a germanium detector. This way, the five different radiative capture transitions which contribute to the ${ }^{14} \mathrm{~N}(\mathrm{p}, \gamma){ }^{15} \mathrm{O}$ cross section at low energy were measured. The total cross section was then studied down to very low energy in the second phase of the experiment by using a $4 \pi$ BGO summing detector placed around a windowless gas target filled with nitrogen at 1 mbar pressure. At the lowest center of mass energy of $70 \mathrm{keV}$ a cross section of 0.24 pbarn was measured, with an event rate of 11 counts/day from the reaction.

The results (fig.3) obtained first with the germanium detector $[12,13]$ and then with the BGO set-up [14] were about a factor two lower than the existing extrapolation $[15,16]$ from previous data $[17,18]$ at very low energy. On the other hand, they were in good agreement with the reanalysis 
[19] of [18] and with the results obtained with indirect methods [20]. Because of this reduction the CNO neutrino yield in the Sun is decreased by about a factor of two.

In order to provide more precise data for the ground state capture, the most difficult one to be measured because of the summing problem, we performed a third phase of the ${ }^{14} \mathrm{~N}(\mathrm{p}, \gamma){ }^{15} \mathrm{O}$ study with a composite germanium detector. This way the total error on the S-factor has been reduced to $8 \%: \mathrm{S}_{1,14}(0)=1.57 \pm 0.13 \mathrm{keV}$ barn [21]. This is significant because, finally solved the solar neutrino problem, we are now facing the solar composition problem: the conflict between helioseismology and the new experimental value of the metal abundance (i.e. the amount of elements different from hydrogen and helium) that emerged from improved modeling of the solar photosphere.

Thanks to the relatively small error, it will be possible in the near future to measure the carbon and nitrogen content of the Sun core by comparing the predicted CNO neutrino flux with the measured one. As a matter of fact, the CNO neutrino flux depends linearly from the carbon and nitrogen abundance and it is decreased by about $30 \%$ in going from the high to the low metallicity scenario [22]. This way it will be possible to test whether the early Sun was chemically homogeneous, a key assumption of the standard Solar Model [23].

The lower cross section is affecting also stars which are more evolved than our Sun. In particular, the lower limit on the age of the Universe inferred from the age of the oldest stellar populations, the globular clusters, is increased by 0.7-1 billion years [24] up to 14 billion years, and thermal pulses during the evolution of asymptotic giant branch (AGB) stars become more powerful, making the dredge-up of carbon to the surface more efficient [25].

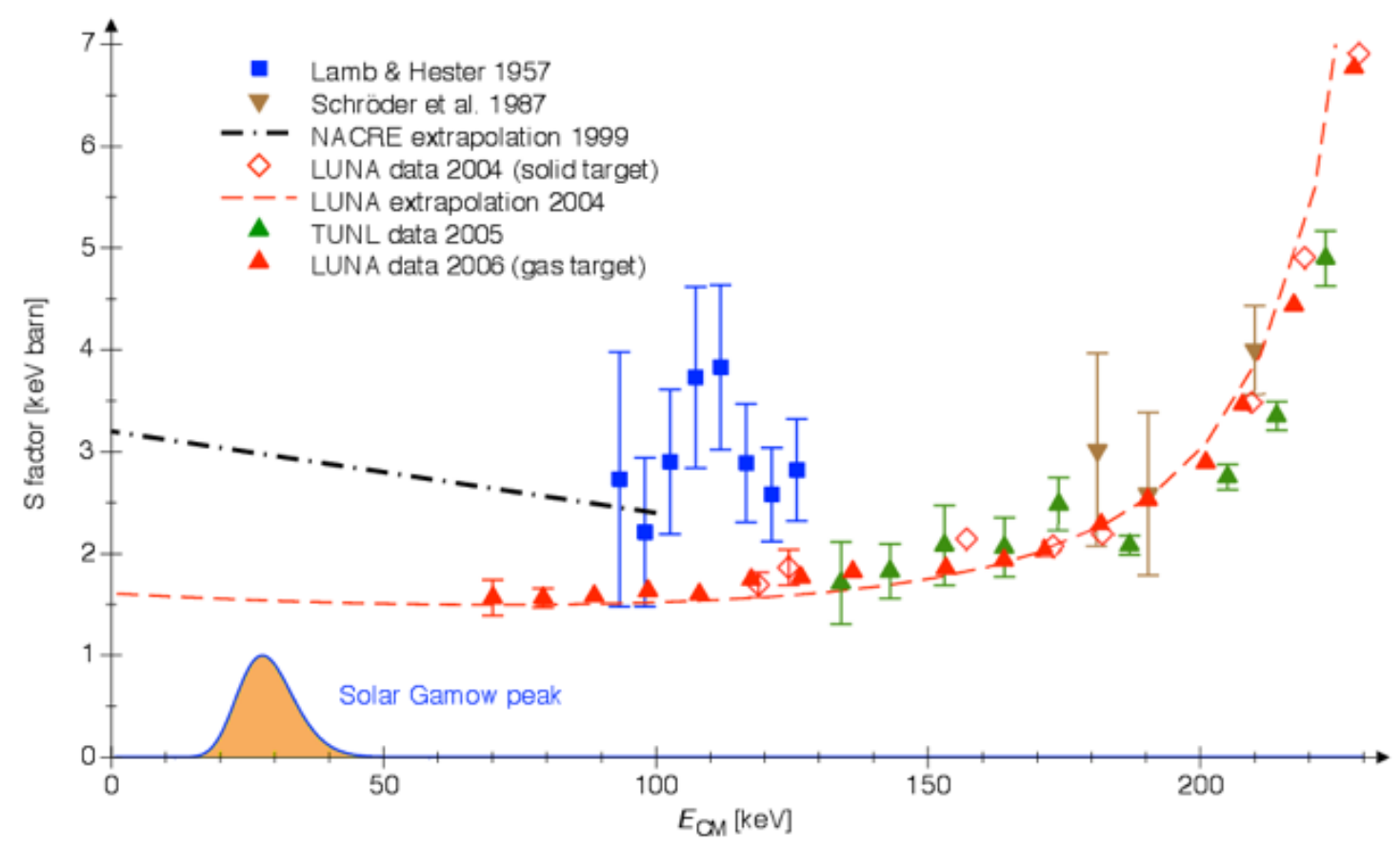

Figure 3: The astrophysical factor of ${ }^{14} N(p, \gamma)^{15} \mathrm{O}$ as function of energy. 


\section{AGB stars, Classical Novae and BBN}

A new and rich program of nuclear astrophysics mainly devoted to $\mathrm{CNO}, \mathrm{Ne}-\mathrm{Na}$ and $\mathrm{Mg}-\mathrm{Al}$ cycles started a few years ago after the solar phase of LUNA. Of particular interest are those bridge reactions which are connecting one cycle to the next, as ${ }^{15} \mathrm{~N}(\mathrm{p}, \gamma){ }^{16} \mathrm{O}[26]$ and ${ }^{17} \mathrm{O}(\mathrm{p}, \gamma){ }^{18} \mathrm{~F}$ [27], the latter competing with ${ }^{17} \mathrm{O}(\mathrm{p}, \alpha){ }^{14} \mathrm{~N}$ [28], or which are key ingredients of gamma astronomy, as ${ }^{25} \mathrm{Mg}(\mathrm{p}, \gamma){ }^{26} \mathrm{Al}[29]$.

Due to the higher Coulomb barrier of the reactions involved, the cycles become important at temperatures higher than the one of our Sun, i.e. during hydrogen burning in the shell of AGB stars and during the thermonuclear runaway of classical Novae (about 30-100, and 100-400 million degrees, respectively). Relatively unimportant for energy generation, these cycles are essential for the 'cooking' of the light nuclei up to ${ }^{27} \mathrm{Al}$. In particular, LUNA is now measuring ${ }^{22} \mathrm{Ne}(\mathrm{p}, \gamma){ }^{23} \mathrm{Na}$ [30], the reaction of the Ne-Na cycle with the highest uncertainty (up to a factor of 2000 in the region of interest due to a large number of predicted resonances), and ${ }^{23} \mathrm{Na}(\mathrm{p}, \gamma){ }^{24} \mathrm{Mg}$, the reaction connecting the $\mathrm{Ne}-\mathrm{Na}$ and $\mathrm{Mg}-\mathrm{Al}$ cycles.

Three low energy resonances of ${ }^{22} \mathrm{Ne}(\mathrm{p}, \gamma)^{23} \mathrm{Na}$ in the energy region of AGB and classical Nova burning have been measured for the first time by LUNA with two germanium detectors [31], giving rise to a reaction rate significantly higher than previous evaluations at temperatures between 80 and 300 millions degrees. A new study of ${ }^{22} \mathrm{Ne}(\mathrm{p}, \gamma)^{23} \mathrm{Na}$ is ongoing at LUNA with a large BGO detector of much higher efficiency to explore smaller strength resonances and to measure the direct capture contribution.

LUNA has achieved significant results also for Big Bang Nucleosynthesis (BBN). In particular, ${ }^{2} \mathrm{H}(\alpha, \gamma)^{6} \mathrm{Li}$ has been measured for the first time in the BBN energy region [32]. The results clearly exclude the cross section enhancement which has been required as nuclear solution to the primordial ${ }^{6} \mathrm{Li}$ problem (i.e. a ${ }^{6} \mathrm{Li}$ abundance in very old stars which seems to be 3 orders of magnitude higher than predicted). The measurement of ${ }^{2} \mathrm{H}(\mathrm{p}, \gamma)^{3} \mathrm{He}$ is currently ongoing underground. This reaction is responsible for the burning of deuterium during BBN. Its $9 \%$ uncertainty in the energy range of interest is the largest uncertainty affecting the prediction of the primordial deuterium abundance. Purpose of the measurement is to reduce the uncertainty on this prediction, about $3 \%$, down to the uncertainty existing on its measurement, almost a factor two smaller.

\section{What next: helium and carbon burning}

After hydrogen burning the natural evolution of LUNA is the study of the next steps in the fusion chain towards ${ }^{56} \mathrm{Fe}$ : helium and carbon burning. In particular, ${ }^{12} \mathrm{C}(\alpha, \gamma){ }^{16} \mathrm{O}$ determines the abundance ratio between carbon and oxygen, the two key elements to the development of life, and it shapes the nucleosynthesis in massive stars affecting the outcomes of both thermonuclear and core-collapse supernovae.

Equally important are ${ }^{13} \mathrm{C}(\alpha, \mathrm{n}){ }^{16} \mathrm{O}$ and ${ }^{22} \mathrm{Ne}(\alpha, \mathrm{n}){ }^{25} \mathrm{Mg}$, the sources of the neutrons which synthesize half of the trans-iron elements through the S-process: neutron capture followed by $\beta$ decay. ${ }^{13} \mathrm{C}(\alpha, \mathrm{n}){ }^{16} \mathrm{O}$ has a Q-value of $2.216 \mathrm{MeV}$ and it operates in the He-burning shell of low mass (less than 4 solar masses) AGB stars. ${ }^{22} \mathrm{Ne}(\alpha, \mathrm{n}){ }^{25} \mathrm{Mg}$ has a negative Q-value (-478 keV) and 
it is active in the He-burning shell of high mass AGB stars and during the core He-burning and the shell C-burning of massive stars (more than 10 solar masses).

Finally, the ${ }^{12} \mathrm{C}+{ }^{12} \mathrm{C}$ fusion reaction is switching on the carbon burning. Its rate determines the evolution of a massive star up to a slowly cooling white dwarf or up to a core-collapse supernova. It also affects the ignition conditions and time scales of thermonuclear supernovae, the standard candles of Cosmology.

This program requires a new 3.5 MV accelerator which is going to be installed underground in the north side of Hall B of Gran Sasso in spring 2018. The new facility will occupy a surface of about $300 \mathrm{~m}^{2}$ (fig.4). The accelerator room will have $80 \mathrm{~cm}$ thick concrete walls and ceiling working as neutron shielding. The maximum neutron flux averaged over the entire external surface of the shielding will be a factor twenty smaller than the LNGS neutron flux, with a similar energy spectrum. The accelerator room will host a single-ended electrostatic accelerator, with two beam lines (only one of them fed at a time) able to deliver $\mathrm{H}, \mathrm{He}^{+}, \mathrm{C}^{+}$and $\mathrm{C}^{2+}$ beams at high current (from $1 \mathrm{~mA}$ of $\mathrm{H}$ to $100 \mathrm{e} \mu \mathrm{A}$ of $\mathrm{C}^{2+}$ ). After the underground mounting of the accelerator and its commissioning, the first physics run will start at the beginning of 2019.

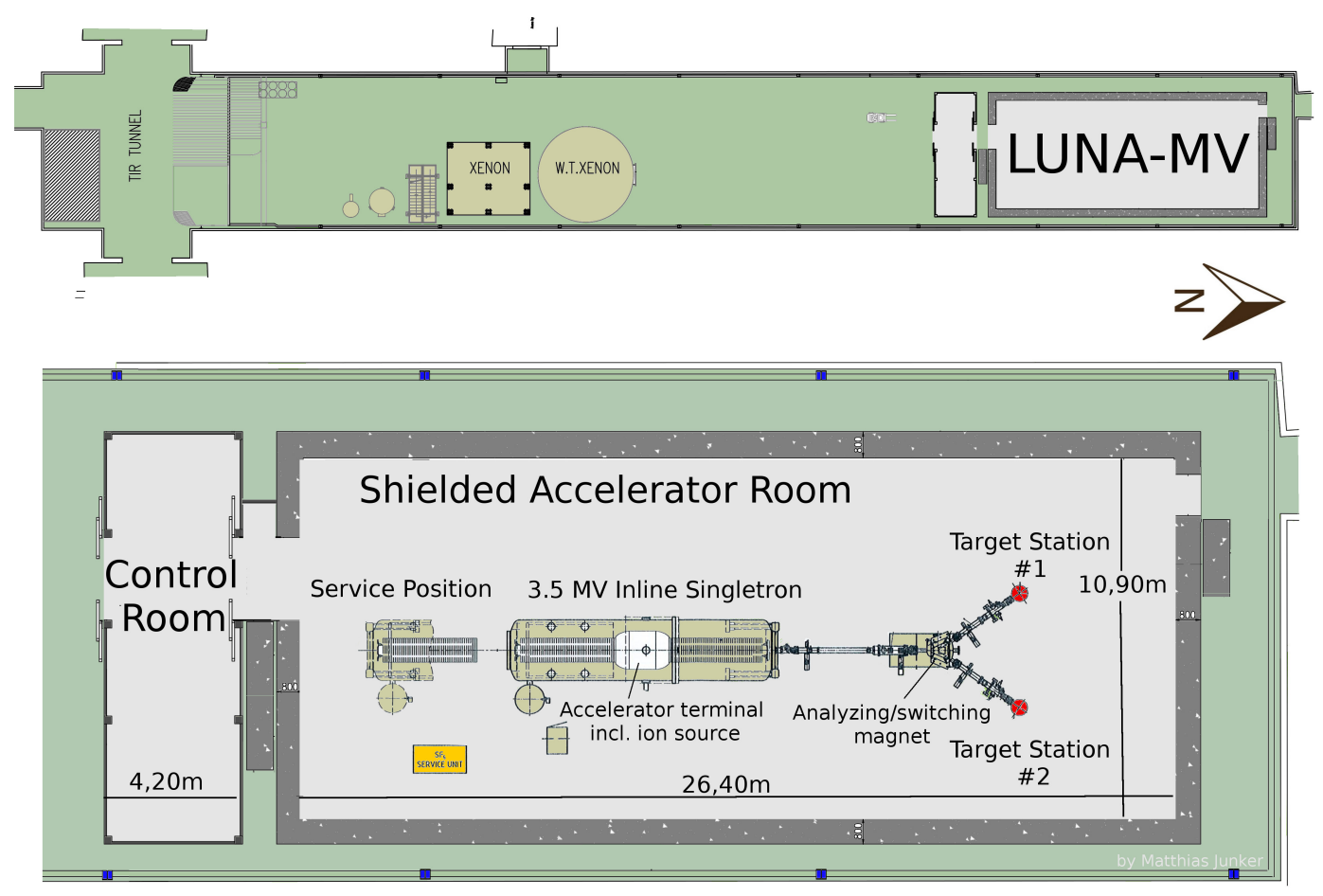

Figure 4: The LUNA-MV facility in the north side of Hall B at LNGS and the 3.5 MV accelerator with the two beam lines.

\section{Conclusions}

Underground nuclear astrophysics was born twenty five years ago in the core of Gran Sasso, with the aim of measuring cross sections in the low energy range and derive reaction rates directly at stellar temperatures. LUNA started its activity as a pilot project with a $50 \mathrm{kV}$ accelerator and still 
remains the only laboratory in the world running an accelerator deep underground, currently a 400 $\mathrm{kV}$ accelerator. The extremely low laboratory background has allowed for the first time nuclear physics experiments with very small count rates, down to a couple of events per month. Only in this way the important reactions responsible for the hydrogen burning in the Sun could be studied down to the relevant stellar energies.

After the solar phase, LUNA is studying the hydrogen burning reactions which are responsible for the 'cooking' of the light elements in AGB stars and classical Novae and is measuring a few key reactions of BBN. The future of LUNA is going to start with the installation of a 3.5 MV accelerator underground in Gran Sasso in spring 2018 and it will be focused on the study of helium and carbon burning in stars.

\section{References}

[1] H. Costantini et al, Rep. Prog. Phys. 72, 086301 (2009).

[2] C. Broggini et al, Annu. Rev. Nucl. Part. Sci. 60, 53 (2010).

[3] U. Greife Greife et al, Nucl. Instrum. Methods A 350, 327 (1994).

[4] A. Formicola et al, (2003), Nucl. Instrum. Methods A 507, 609 (2003).

[5] D. Bemmerer, Eur. Phys. J. A 24, 2 (2005).

[6] G. Bellini, C. Broggini, A. Guglielmetti, Eur. Phys. J. A 52, 88 (2016).

[7] R. Bonetti et al, Phys. Rev. Lett. 82, 5205 (1999).

[8] A. Krauss et al, Nucl. Phys. A467, 273 (1987).

[9] M. Dwarakanath and H. Winkler, Phys. Rev. C 4, 1532 (1971).

[10] N. Kudomi et al, Phys. Rev. C 69, 015802 (2004).

[11] F. Confortola et al, Phys. Rev. C 75, 065803 (2007).

[12] C. Formicola et al, Phys. Lett. B 591, 61 (2004).

[13] G. Imbriani et al, Eur. Phys. J. A 25, 455 (2005).

[14] A. Lemut et al, Phys. Lett. B 634, 483 (2006).

[15] E.G. Adelberger et al, Rev. Mod. Phys. 70, 1265 (1998).

[16] C. Angulo et al, Nucl. Phys. A 656, 3 (1999).

[17] W.A.S. Lamb and R.E. Hexter, Phys. Rev. 108, 1304, (1957).

[18] U. Schroder et al, Nucl. Phys. A 467, 240 (1987).

[19] C. Angulo and P. Descouvement, Nucl. Phys. A 690, 755 (2001).

[20] A.M. Mukhamedzhanov et al, Phys. Rev. C 67, 065804 (2003).

[21] M. Marta et al, Phys. Rev. C 78, 022802 (2008).

[22] A.M. Serenelli, Eur. Phys. J. A 52, 78 (2016).

[23] W.C. Haxton and A.M. Serenelli, Astrophysical Journal 687, 678 (2008). 
[24] G. Imbriani et al, Astronomy and Astrophysics 420, 625 (2004).

[25] F. Herwig and S.M. Austin, Astrophysical Journal 613, L73 (2004).

[26] A. Caciolli et al, Astronomy and Astrophysics 533, A66 (2011).

[27] D.A. Scott et al, Phys. Rev. Lett. 109, 202501 (2012).

[28] C. G. Bruno et al, Eur. Phys. J. A 51, 94 (2015).

[29] O. Straniero et al, Astrophysical Journal 763, 100 (2013).

[30] F. Cavanna et al, Eur. Phys. J. A 50, 179 (2014).

[31] F. Cavanna et al, Phys. Rev. Lett. 115, 252501 (2015).

[32] M. Anders et al, Phys. Rev. Lett. 113, 042501 (2014).

\section{DISCUSSION}

JIM BEALL: What will the stability be in energy for the $3.5 \mathrm{MV}$ accelerator?

CARLO BROGGINI: The beam energy stability over 1 hour will be $1 \times 10^{-5} \times \mathrm{TV}$ or $20 \mathrm{~V}$, whichever is higher (TV is the terminal voltage).

JONATHAN TAN: Now that you have revised globular cluster age up by about $1 \mathrm{~Gy}$, what is the effect which sets the uncertainty on these age estimates?

CARLO BROGGINI: There are three main uncertainties: the globular cluster distance, its metallicity and the light extinction along the line of sight. 